\title{
Problématiques des hydrocarbures aromatiques polycycliques dans les corps gras
}

Oléagineux, Corps Gras, Lipides. Volume 10, Numéro 4, 287-95, JUILLET-AOÛT 2003, Problématiques actuelles dans le domaine de l'analyse des oléagineux et des corps gras

Auteur(s) : Florence LACOSTE, Renée RAOUX, Dorothée DUBOIS, Bénédicte SOULET, Institut des Corps Gras, ITERG, Rue Monge, 33600 Pessac, France <f.lacosteiterg.com> .

Author(s) : Florence LACOSTE, Renée RAOUX, Dorothée DUBOIS, Bénédicte SOULET

Résumé : La présence des hydrocarbures aromatiques polycycliques (HAP) dans les huiles végétales peut provenir de différentes sources, mais généralement les huiles contaminées sont issues de matières premières nécessitant d'être séchées telles que les pépins de raisin, le coprah ou les grignons d'olive. Actuellement, il n'y a pas de réglementation européenne limitant la teneur en HAP dans les produits alimentaires mais un Code de Bonnes Pratiques a été établi par FEDIOL ${ }^{1}$. La détermination de la totalité des HAP présents dans les corps gras est rendue délicate par le faible niveau de chaque PAH à quantifier et la complexité des étapes d'extraction et de purification. La seule méthode faisant l'objet d'une norme (NF EN ISO 15302) concerne la détermination de la teneur en benzo[ $a$ ]pyrène. Un projet de méthode fourni par I'ITERG est en cours d'évaluation dans le cadre des travaux du Sous-Comité ISO\\TC34\\SC11 des "Corps gras d'origines animale et végétale ". Cette méthode permet la quantification de 12 HAP dans les corps gras, avec une limite de quantification de 0,2 $\mu \mathrm{g} \backslash \backslash \mathrm{kg}$ pour tous les composés analysés, à l'exception des fluoranthène et benzo[ $g, h, i]$ pérylène où celle-ci est de $0,3 \mu \mathrm{g} \backslash \backslash \mathrm{kg}$, ainsi que de l'indéno[ 1,2,3-cd]pyrène pour lequel la limite de quantification atteint $1 \mu \mathrm{g} \backslash \backslash \mathrm{kg}$. Depuis quatre ans, les travaux de I'ITERG sur les HAP ont été centrés sur l'optimisation et la normalisation d'une procédure d'analyse (ISO \ICD 15753), sur l'estimation du niveau de contamination des huiles végétales produites en France et sur l'effet du raffinage sur le niveau en HAP. Chaque étape de ce travail est développée ici.

Summary : The presence of polycyclic aromatic hydrocarbons (PAHs) in vegetable oils may be due to different sources, but generally the contaminated oils come from raw material that need to be dried, such as grape seeds, coconut, or pomace olive. At the present time, there is no European regulation for PAHs but an Industrial Code of Practice was provided by FEDIOL which is the European Association of Oilseed Crushers. PAHs determination in fatty material is difficult mainly due to low level of individual PAHs to be quantified and to the complexity of the extraction and purification steps. The only test method already standardised (NF EN ISO 15302) deals with the determination of benzo[ a]pyrene. A committee draft test method provided by ITERG is under evaluation within ISO \ITC34\\SC11 for determination of 12 PAHs. Limits of quantification are respectively $1 \mu \mathrm{g} \backslash \backslash \mathrm{kg}$ for indeno[ 1,2,3-cd] pyrene, $0.3 \mu \mathrm{g} \backslash \backslash \mathrm{kg}$ for fluoranthene and benzo[ $g, h, i]$ perylene, $0,2 \mu \mathrm{g} \backslash \backslash \mathrm{kg}$ for 9 other compounds analysed. For the last 4 years, ITERG's work on PAHs was focused on improvement and standardisation of the analytic procedure (ISO IICD 15753), estimation of level of contamination of French vegetable oils (mainly grapeseed oils) and the evaluation of refining effect on the PAHs contamination. Each part of this work is summarised in this paper. 
Mots-clés : hydrocarbures aromatiques polycycliques, huiles végétales, réglementation, dosage, corrélation

Keywords : polycyclic aromatic hydrocarbons, vegetable oil, regulation, determination, correlation

\section{ARTICLE}

Auteur(s) : Florence LACOSTE, Renée RAOUX', Dorothée DUBOIS, Bénédicte SOULET

Institut des Corps Gras, ITERG, Rue Monge, 33600 Pessac, France

<f.lacoste@iterg.com>

Les hydrocarbures aromatiques polycycliques (HAP) sont des contaminants universels de notre environnement et de la chaîne alimentaire de I'homme. La présence des HAP dans les huiles végétales peut provenir de différentes sources telles que la pollution atmosphérique, le passage via la plante à partir de sols contaminés ou le séchage direct des graines par des gaz de combustion.

En raison de la contribution notable des huiles végétales dans la consommation en HAP et du probable pouvoir cancérogène de ces composés, les HAP sont considérés actuellement comme un problème de sécurité sanitaire par les producteurs de corps gras. Avant de suivre la contamination des huiles et graisses, il convient de répondre aux questions suivantes :

- Quels sont les composés devant être impérativement dosés ?

- Quelles sont les réglementations à respecter?

- Existe-t-il une méthode d'analyse normalisée?

L'objectif est ici de fournir des réponses à ces questions. L'évaluation du raffinage sur la concentration en HAP et les résultats d'une enquête sur la contamination des huiles végétales produites en France viennent compléter cet article.

\section{Structure chimique et formation des hydrocarbures aromatiques polycycliques}

Les hydrocarbures aromatiques polycycliques sont composés de 2 à 6 cycles aromatiques condensés. Le tableau 1 présente la structure chimique de 16 HAP faisant partie d'une liste établie par I'US-EPA 1 en 1984 [1]. Une distinction entre les " HAP légers " possédant de 2 à 4 cycles et les " HAP lourds " possédant plus de 4 cycles est souvent faite au niveau des ouvrages traitant du sujet. La formation des HAP apparaît lors de la combustion incomplète ou de la pyrolyse de matière organique comme le bois, les produits pétroliers, le charbon ou les aliments. Elle peut également avoir lieu à basse température $\left(100-150^{\circ} \mathrm{C}\right)$ avec cependant une cinétique très lente. Les origines de la présence des HAP dans les produits alimentaires sont principalement liées à l'environnement ou à des procédés technologiques. En effet, ces composés sont présents dans les émissions industrielles, les émissions liées au chauffage domestique et au transport, ainsi que dans celles des stations d'incinération de déchets. Les cultures peuvent alors être contaminées directement par l'air mais aussi indirectement par le sol ou l'eau après dépôt puis transfert. Les procédés technologiques tels que le fumage, le grillage, le séchage et la torréfaction des aliments génèrent également des 
quantités de HAP non négligeables. Les groupes d'aliments connus pour leur contribution à l'apport alimentaire de HAP sont les viandes grillées, les céréales, les légumes et les corps gras [2-5]. En ce qui concerne les corps gras, la contamination est principalement due au séchage direct des graines par des gaz de combustion en l'absence d'échangeur de chaleur, au cours duquel les produits de combustion entrent en contact avec la matière première. C'est le cas notamment des pépins de raisin, du coprah ou des grignons d'olive qui nécessitent d'être séchés avant l'extraction de l'huile.

${ }^{1}$ Agence de Protection de l'Environnement des Etats-Unis

\section{Toxicité des hydrocarbures aromatiques polycycliques}

Un avis sur la toxicité des HAP a été émis il y a vingt ans par le Centre International de la Recherche sur le Cancer [6]. Les trois composés ayant été jugés les plus toxiques car présentant un pouvoir cancérogène probable (groupe $2 \mathrm{~A}$ ) sont le benzo[a]pyrène (BaP), le benzo[ $a]$ anthracène $(\mathrm{BaA})$ et le dibenzo[a,h]anthracène (DBahA). Vis-à-vis de ce pouvoir carcinogène, quatre autres composés présentent un risque possible (groupe 2B). II s'agit des benzo[b]fluoranthène (BbFA), benzo[j]fluoranthène (BjFA), benzo[k]fluoranthène (BkFA) et indéno[1,2,3,cd]pyrène (IP). Sept autres composés ont été jugés inclassables concernant le risque encouru. Selon cet avis, il serait donc important de concentrer l'effort analytique sur les sept composés jugés toxiques. En 1992, NISBET et LAGOY [7] ont proposé d'introduire des facteurs d'équivalence en toxicité, appelés plus génériquement TEF, afin de pondérer la quantification des HAP totaux par leur niveau de toxicité. Les facteurs d'équivalence de toxicité utilisés pour les HAP sont basés sur leur effet carcinogène et non pas sur leur effet au niveau du métabolisme cellulaire comme pour les dioxines. Le BaP étant admis comme le HAP le plus toxique, il a été choisi comme composé de référence avec un TEF arbitrairement fixé à 1. La liste initialement proposée contenait 16 congénères (tableau 2) avec 9 composés ayant des TEF compris entre 5 et 0,001: BaP, DBahA, BaA, BbFA, BkFA, IP, benzo[ $g, h, i]$ pérylène (BghiP), anthracène (ATR) et chrysène (CHR). L'utilisation des TEF est la suivante: la concentration de chaque HAP individuel est multipliée par la valeur du TEF puis la somme de ces concentrations pondérées est exprimée en $\mu \mathrm{g} / \mathrm{kg}$ d'équivalent BAP (BAPEQ). Cette pondération permet de prendre en compte majoritairement les composés les plus toxiques et d'éventuellement comparer des résultats, même si les listes de composés quantifiés diffèrent légèrement.

Tableau 2. Liste des facteurs toxiques équivalents pour les hydrocarbures polycycliques aromatiques proposée par Nisbet et Lagoy (1992) et par I'AFSSA. Opinion de la Commission Européenne pour la génotoxicité des hydrocarbures polycycliques aromatiques.

\begin{tabular}{l|l|l|l}
\multicolumn{1}{l}{$\begin{array}{l}\text { génotoxique } \\
\text { benzo[ } b] \text { fluoranthène }\end{array}$} & 0,1 & 0,1 & génotoxique \\
\hline benzo[j]fluoranthène & & 0,1 & génotoxique \\
\hline benzo[k]fluoranthène & 0,1 & 0,1 & génotoxique \\
\hline indéno[1,2,3-cd]pyrène & 0,1 & 0,1 & génotoxique
\end{tabular}




\begin{tabular}{|l|l|l|l|}
\hline $\begin{array}{l}\text { benzo[a]pyrène } \\
\text { dibenzo[a,h]anthracène }\end{array}$ & 1 & 1 & génotoxique \\
\hline cyclopenta[c,d]pyrène & 5 & 1 & génotoxique \\
\hline dibenzo[a,e]pyrène & & génotoxique \\
\hline dibenzo[a,h]pyrène & génotoxique \\
\hline dibenzo[a,i]pyrène & génotoxique \\
\hline dibenzo[a,I]pyrène & & génotoxique \\
\hline 5-méthylchrysène & & génotoxique \\
\hline
\end{tabular}

$\mathrm{L}^{\prime} A F S S A^{\underline{2}}$ a récemment choisi d'abaisser la valeur du TEF du DBahA de 5 à 1 , ce qui est tout à fait en accord avec le fait que le BaP est généralement considéré comme le composé le plus toxique.

A la fin de l'année 2002, le Comité Scientifique Européen sur les Aliments a publié un rapport très complet [5] sur l'évaluation du risque lié à la présence des HAP dans les aliments. A la lueur d'études in vivo récentes, quinze composés sur 33 ont été jugés comme ayant une action mutagène/génotoxique avérée (tableau 2). Outre les 7 composés déjà cités, il apparaît dans cette liste six composés jamais recherchés jusqu'à présent dont 4 isomères du dibenzopyrène, le cyclopenta[cd]pyrène et le 5 -méthylchrysène.

Au lieu de promouvoir l'utilisation des TEF pour l'évaluation du risque lié aux HAP, le Comité recommande l'utilisation du benzo[a]pyrène comme un marqueur de la présence des HAP dans les aliments. Le pouvoir carcinogène des HAP totaux est évalué à environ dix fois celui du BaP.

L'ensemble de ces informations peut apparaître assez conflictuel pour un industriel qui doit assurer un contrôle du niveau de HAP dans une huile végétale. Quelle liste de composés choisir ? Faut-il utiliser les facteurs d'équivalence de toxicité ? Malheureusement, l'examen de la réglementation ne fournit pas une aide décisive.

${ }^{2}$ Agence pour la Sécurité Sanitaire des Aliments

\section{Réglementations relatives aux hydrocarbures aromatiques polycycliques}

Actuellement, il n'y a pas de réglementation européenne concernant les HAP. Le rapport du Comité Scientifique Européen sur les Aliments [5] recommande que l'exposition des aliments soit aussi faible que raisonnablement possible (ALARA). Heureusement, un code des bonnes pratiques a été proposé par FEDIOL dès 1993. II fixe une limite supérieure pour la teneur en HAP totaux à $25 \mu \mathrm{g} / \mathrm{kg}$ (13 composés), ainsi qu'une limite supérieure pour la teneur en HAP lourds à $5 \mu \mathrm{g} / \mathrm{kg}$ (BaP, DBahA, BghiP, BeP, pérylène, anthanthrène, coronène). II stipule également que lorsque la teneur en benzo[ $a$ ]pyrène dans une huile brute est supérieure à $1 \mu \mathrm{g} / \mathrm{kg}$, un traitement sur charbon actif durant le raffinage est nécessaire. Récemment, une réglementation a été mise en place en Italie et 
en Espagne suite à la mise en évidence de la contamination en HAP des huiles de grignons d'olive. Elle concerne sept HAP lourds connus pour leur toxicité (BaP, DBahA, BaA, BbFA, BkFA, IP, BghiP) ainsi qu'un autre congénère peu souvent mentionné, le benzo[e]pyrène (BeP). Chaque HAP est limité à $2 \mu \mathrm{g} / \mathrm{kg}$ et leur somme doit être inférieure ou égale à $5 \mu \mathrm{g} / \mathrm{kg}$. Pour ces différentes recommandations ou réglementations, les facteurs équivalents toxiques ne sont pas utilisés. Néanmoins, certains pays comme le Canada les mettent en pratique. Le Canada applique une limite de $3 \mu \mathrm{g} / \mathrm{kg}$ pour la teneur totale en HAP dans les huiles de grignons d'olive (BaP, BaA, DBahA, BbFA, $B k F A, I P, C H R, B g h i P)$, exprimée en équivalent BaP et dont le calcul est basé sur les TEF donnés par les lignes directrices de l'OMS ${ }^{3}$. Notre position est, dans l'attente d'une réglementation européenne, de suivre le code de bonnes pratiques de FEDIOL qui est généralement appliqué par les industriels de I'huilerie, en utilisant une liste de HAP lourds limitée aux composés suivants: BaP, DBahA, BbFA, BkFA, IP, BghiP.

${ }^{3}$ Organisation Mondiale de la Santé

\section{Analyse des HAP et normalisation}

La détermination des HAP dans les corps gras est de l'analyse de traces. Les principales difficultés sont liées à :

- la difficulté d'extraire, isoler et purifier les HAP qui sont très lipophiles, - le niveau de concentration très faible de chaque HAP à quantifier $(\mu \mathrm{g} / \mathrm{kg})$. La maîtrise de paramètres tels que la pureté des solvants utilisés, l'efficacité de l'étape d'extraction, la limitation des étapes d'évaporation, la résolution chromatographique et la sensibilité du détecteur, est essentielle pour obtenir des résultats fiables. La seule méthode faisant actuellement l'objet d'une norme, l'ISO 15302 [8], concerne la détermination du benzo[a]pyrène qui est considéré comme un marqueur de la présence des HAP. Le protocole décrit dans la figure 1 est très simple : dilution de l'échantillon dans un solvant apolaire, fractionnement par extraction sur phase solide (SPE) à l'aide d'une colonne d'alumine, élution des HAP avec de l'éther de pétrole, évaporation à sec et analyse par chromatographie liquide haute performance (HPLC) sur une colonne de phase inverse reliée à un détecteur à fluorescence. La figure 2 présente le chromatogramme d'un échantillon d'huile raffinée contenant $7 \mu \mathrm{g} / \mathrm{kg}$ de BaP, et le tracé montre que d'autres composés peuvent induire une co-élution. Une légère modification de la méthode, récemment proposée par un industriel français, permet le dosage en routine de six HAP lourds (BaP, DBahA, BbFA, BkFA, IP, BghiP) dans les matrices simples comme les huiles brutes de tournesol [9]. Le rapport du Comité Scientifique Européen sur les Aliments [5] conclut que même si le BaP peut être considéré comme un marqueur, il est nécessaire de poursuivre la collecte de données sur la totalité du profil des HAP afin d'évaluer la contamination des aliments. Pour atteindre cet objectif, une méthode reconnue permettant le dosage de tous les HAP présents dans I'huile est essentielle. Différents protocoles publiés récemment portent sur la détermination des congénères de HAP dans les corps gras. La préparation de l'échantillon fait intervenir une extraction liquide/liquide suivie d'une purification sur : i) une colonne de silice [10], ii) une colonne de silice puis une colonne de silice greffée amino-propyle [11], iii) une colonne de chromatographie d'exclusion stérique [12], iv) une colonne de chromatographie basée sur un complexe donneur-accepteur [13]. En 1999, I'ITERG a développé une méthode de détermination de $12 \mathrm{HAP}$, dont 6 composés légers et 6 lourds. La préparation de l'échantillon est basée sur un protocole déjà utilisé pour les polychlorobiphényles [14]. II consiste en trois étapes 
successives : pré-concentration des HAP dans la matrice, extraction des HAP de l'huile résiduelle et purification sur une cartouche de silice greffée C18 et enfin, sur une cartouche de Florisil (figure 3A). L'analyse est réalisée par HPLC avec un détecteur à fluorescence (tableau 3). Lors du développement de la méthode, les différents paramètres ont été optimisés afin d'obtenir des taux de récupération répétables et suffisamment élevés tout en limitant le volume de solvant utilisé (150 $\mathrm{ml})$. Les taux de récupération sont supérieurs à $70 \%$ pour 11 des composés (PHE, ATR, FA, PY, BaA, CHR, BbFA, BkFA, $\mathrm{BaP}, \mathrm{IP}, \mathrm{DBahA})$ et entre $60 \%$ et $70 \%$ pour le BghiP. La limite de quantification est de $0,2 \mu \mathrm{g} / \mathrm{kg}$ à l'exception des fluoranthène et benzo[g,h,i]pérylène $(0,3 \mu \mathrm{g} / \mathrm{kg})$, et de l'indéno[1,2,3,cd]pyrène $(1 \mu \mathrm{g} / \mathrm{kg})$.

Tableau 3. Procédure développée par l'Iterg pour la détermination des hydrocarbures polycycliques aromatiques dans les huiles végétales (ISO/CD 15753 : 2001). Conditions HPLC.

\begin{tabular}{l|l|l|}
\hline Débit : $1,2 \mathrm{ml} / \mathrm{min}$ & Volume injecté $: 20 \mu \mathrm{l}$ Température de colonne $: 25^{\circ} \mathrm{C}$ \\
\hline Temps (min) & Acétonitrile (\%) & Acétonitrile/eau $(50: 50 \mathrm{v} / \mathrm{v})(\%)$ \\
\hline 5 & 0 & 100 \\
\hline 27 & 0 & 100 \\
\hline 36 & 60 & 40 \\
\hline 41 & 100 & 0 \\
\hline 43 & 100 & 0 \\
\hline 45 & 0 & 100 \\
\hline
\end{tabular}

\begin{tabular}{|c|c|c|c|c|}
\hline Groupe & Composé & Temps (min) & Excitation (nm) & Emission (nm) \\
\hline 1 & $N A, A C, F L$ & 0 & 270 & 324 \\
\hline 2 & PHE, ATR & 12,6 & 248 & 375 \\
\hline 3 & FA & 16,4 & 280 & 462 \\
\hline 4 & PY, BaA, CHR & 18,05 & 270 & 385 \\
\hline 5 & $\mathrm{BbFA}$ & 28,0 & 256 & 446 \\
\hline
\end{tabular}




\begin{tabular}{|c|c|c|c|c|}
\hline 6 & BkFA, BaP, DBahA, BghiP & 31,1 & 292 & 410 \\
\hline 7 & IP & 38,0 & 274 & 507 \\
\hline
\end{tabular}

Naphtalène (NA), Acénaphtène $(A C)$, Fluorène $(F L)$, Phénanthrène (PHE), Anthracène (ATR), Fluoranthène ( $\mathrm{FA})$, Pyrène (PY), Benzo $[a]$ anthracène $(\mathrm{BaA})$, Chrysène $(\mathrm{CHR})$, Benzo[b]fluoranthène $(\mathrm{BbFA})$, Benzo[k]fluoranthène (BkFA), Benzo[a]pyrène (BaP), Dibenzo[a,h]anthracène (DBahA), Benzo $[g, h, i]$ pérylène (BghiP), Indéno[1,2,3-cd]pyrène (IP).

Ce protocole peut être utilisé pour les huiles végétales (colza, tournesol, soja, pépins de raisin, olive) mais il ne convient pas pour les huiles de coprah en raison de la présence d'acides gras à courtes chaînes. Une modification de la méthode adaptée à l'huile de coprah a été également développée, en réduisant la prise d'essai, en utilisant deux cartouches de silice greffée C18 et deux cartouches de Florisil et en modifiant la composition du solvant d'élution (figure $3 B$ ). Les taux de récupération sont alors supérieurs à $70 \%$ pour huit composés (PHE, ATR, FA, PY, BaA, CHR, BbFA, BkFA), entre $60 \%$ et $70 \%$ pour le benzo[a]pyrène et le dibenzo[a,h]anthracène, et entre $40 \%$ et $50 \%$ pour le benzo[g,h,i]pérylène et l'indéno[1,2,3,cd]pyrène. La figure 4 présente un chromatogramme d'une huile raffinée de tournesol complémentée avec $1 \mu \mathrm{g} / \mathrm{kg}$ de chaque HAP. Elle montre que les HAP légers tels que le naphtalène, l'acénaphtène et le fluorène sont partiellement perdus lors l'évaporation du solvant et ne peuvent être quantifiés. La sensibilité est correcte pour la plupart des composés à l'exception de l'indéno[1,2,3,cd]pyrène où la limite de quantification est évaluée à $1 \mu \mathrm{g} / \mathrm{kg}$. La figure 5 fournit un chromatogramme d'une huile de palme raffinée et la compare à une solution de référence contenant $5 \mu \mathrm{g} / \mathrm{kg}$ par PAH. Quelques pics dus à la matrice apparaissent et peuvent interférer avec le benzo[a]anthracène et le dibenzo[a,h]anthracène. La figure 6 illustre la difficulté d'analyser une huile raffinée de grignons d'olive en raison de nombreuses interférences. En comparant les spectres d'émission et d'excitation des pics à ceux des substances de référence, le seul composé identifié est le chrysène. Cette méthode a été proposée au sous-comité ISO/TC34/SC11 des "Corps gras d'origines animale et végétale » en 2001 et est actuellement en évaluation pour en déterminer la fidélité [15].

\section{Effet du raffinage sur la contamination en HAP}

Une étude relative à l'élimination des HAP au cours du raffinage a été réalisée pour trois échantillons d'huile brute de pépins de raisin fournis par un industriel français. Le raffinage comprend différentes étapes : neutralisation, décoloration et désodorisation (figure 7). Afin de réduire la teneur en HAP, la décoloration a été conduite à $100^{\circ} \mathrm{C}$ sous vide avec un mélange de terre décolorante et de charbon actif. La neutralisation est une étape qui réduit faiblement la teneur en HAP, puisque seulement $25 \%$ des HAP légers et lourds sont éliminés. Ajouter du charbon actif pendant l'étape de décoloration permet d'éliminer $85 \%$ des HAP légers restants et la totalité des HAP lourds. La désodorisation est également une étape efficace pour la réduction des HAP légers; en effet, le rendement d'élimination est de $94 \%$. L'ensemble de ces résultats prouve que les HAP lourds sont totalement éliminés lors de l'étape de décoloration alors que les HAP légers disparaissent pendant la décoloration et la désodorisation. 


\section{Niveau de la contamination des huiles raffinées françaises}

Quarante-deux échantillons d'huile raffinée ont été analysés à l'aide de la méthode développée par I'ITERG. Les échantillons ont été collectés auprès d'industriels ou en supermarchés en 1999 et 2000, soit 12 huiles de pépins de raisin, 12 huiles de tournesol, 7 huiles de colza, 5 huiles de coprah, 2 huiles de soja, 1 huile d'arachide, 1 huile de maïs et 2 huiles de palme (tableau 4). Comme il n'existe pas de réglementation européenne, les résultats ont été jugés en fonction du code de bonnes pratiques de FEDIOL qui limite la teneur en HAP lourds à $5 \mu \mathrm{g} / \mathrm{kg}$ et la teneur en benzo[a]pyrène à $1 \mu \mathrm{g} / \mathrm{kg}$. Les HAP lourds (BaP, DBahA, BbFA, BkFA, IP, BghiP) ont été sommés pour comparer leur total à la limite fixée par FEDIOL.

Tableau 4. Etude de la contamination en hydrocarbures polycycliques aromatiques des huiles végétales raffinées en France.

\begin{tabular}{|c|c|c|c|c|c|}
\hline Tournesol & Colza & $\begin{array}{l}\text { Pépins de } \\
\text { raisin }\end{array}$ & Coprah & Huiles raffinées & \\
\hline $\begin{array}{l}\mathrm{Nb} \\
\text { d'échantillons }\end{array}$ & 12 & 7 & 12 & 5 & 42 \\
\hline Somme $\leq 5$ & $\begin{array}{l}83 \% \text { des } \\
\text { échantillons }\end{array}$ & $\begin{array}{l}100 \% \text { des } \\
\text { échantillons }\end{array}$ & $\begin{array}{l}67 \% \text { des } \\
\text { échantillons }\end{array}$ & 3 échantillons & $\begin{array}{l}79 \% \text { des } \\
\text { échantillons }\end{array}$ \\
\hline Somme $>5$ & $\begin{array}{l}2 \text { échantillons } \\
(5,2-33,6)\end{array}$ & - & $\begin{array}{l}4 \text { échantillons } \\
(9,3 \text { à } 14,8)\end{array}$ & $\begin{array}{l}2 \text { échantillons } \\
(5,3-19,2)\end{array}$ & $\begin{array}{l}21 \% \text { des } \\
\text { échantillons }\end{array}$ \\
\hline
\end{tabular}

Les huiles raffinées de pépins de raisin sont souvent en-dehors des limites fixées par FEDIOL. Sept échantillons sur 12 analysés ont une teneur en BaP supérieure à $1 \mu \mathrm{g} / \mathrm{kg}$, mais seulement 4 d'entre eux ont une teneur en HAP lourds supérieure à $5 \mu \mathrm{g} / \mathrm{kg}$. II paraît donc, à première vue, plus fiable d'utiliser une méthode quantifiant 12 HAP plutôt qu'une extrapolation de la teneur en HAP lourds en multipliant la teneur en HAP par un facteur de 5 . Une seule huile raffinée de tournesol présente une teneur élevée en HAP lourds $(33,6 \mu \mathrm{g} / \mathrm{kg})$, ce qui est cohérent avec la teneur élevée en $\mathrm{BaP}$ $(8,6 \mu \mathrm{g} / \mathrm{kg})$. Cette valeur élevée montre que I'utilisation du charbon actif pendant l'étape de décoloration n'est pas encore systématiquement mise en œuvre. Deux huiles raffinées de coprah sur les 5 analysées contiennent plus de HAP lourds que la limite de FEDIOL, respectivement 5,3 $\mu \mathrm{g} / \mathrm{kg}$ et $19,2 \mu \mathrm{g} / \mathrm{kg}$. Ces résultats confirment que le coprah est une matière première qui présente un risque élevé de contamination. Les huiles de colza, arachide, maïs et soja sont faiblement contaminées. Seulement un des 11 échantillons analysés contient une teneur en HAP lourds supérieure à la limite fixée (soja : 7,4 $\mu \mathrm{g} / \mathrm{kg}$ ). Vingt-et-un pour cent de ces échantillons ont une teneur en hydrocarbures aromatiques polycycliques supérieure à $5 \mu \mathrm{g} / \mathrm{kg}$, la limite fixée par FEDIOL, soit 9 échantillons sur 42 . Les matières à risque sont les pépins de raisin et le coprah en raison de la nécessité de les sécher avant d'extraire l'huile. 


\section{Corrélation entre benzo[ $a]$ pyrène et les HAP lourds}

Une base de données de 62 échantillons a été constituée, à partir des résultats présentés précédemment ainsi que de ceux collectés pour des huiles brutes de pépins de raisin, afin d'évaluer la corrélation respectivement entre le benzo[a]pyrène et les HAP totaux, et le BaP et les HAP lourds. $\mathrm{BaP}$, HAP lourds et HAP totaux ont été déterminés en utilisant la méthode de I'ITERG. L'objet de cette étude était de vérifier que les rapports entre BaP et HAP totaux, ainsi que BaP et HAP lourds, étaient comparables aux valeurs données par le code de FEDIOL. En effet, si ces ratios étaient suffisamment fiables, il serait moins contraignant pour les industriels de ne doser que le benzo[ $a$ ]pyrène plutôt que la totalité des HAP lors $d u$ contrôle de la qualité des huiles raffinées. La corrélation entre le BaP et les HAP totaux est nette mais le coefficient de corrélation $(0,76)$ n'est pas très bon en raison d'une huile brute de pépins de raisin présentant une forte concentration en phénanthrène, fluoranthène et pyrène pour une relativement faible teneur en BAP (figure 8). Le facteur de proportionnalité entre BaP et HAP totaux est ici de 27 , ce qui est très proche du ratio de 25 donné par FEDIOL. La corrélation entre le BaP et les HAP lourds est bien meilleure avec un coefficient de corrélation de 0,96 et un facteur de proportionnalité proche de 4 (figure 9). Utiliser la limite en BaP de $1 \mu \mathrm{g} / \mathrm{kg}$ au lieu de la limite à $5 \mu \mathrm{g} / \mathrm{kg}$ en HAP lourds pose un problème uniquement dans le cas où la teneur en BaP est comprise entre $1 \mu \mathrm{g} / \mathrm{kg}$ et $2 \mu \mathrm{g} / \mathrm{kg}$. En effet dans ce cas, il peut arriver de considérer des échantillons comme " contaminés » alors que leur teneur en HAP lourds ne dépasse pas réellement la valeur de $5 \mu \mathrm{g} / \mathrm{kg}$ (tableau 5).

Tableau 5. Base de données utilisée pour établir la corrélation entre le benzo[a]pyrène et les hydrocarbures polycycliques aromatiques lourds.

\begin{tabular}{l|l|l|l|l|}
$\begin{array}{l}\text { Nombre total } \\
\text { d'échantillons }\end{array}$ & \multicolumn{3}{l}{ Nombre d'échantillons avec } & \\
\cline { 2 - 4 } & BAP $>1$ & HAP lourds $>5$ & HAP totaux $>25$ & \\
\hline BAP entre 0 et 1 & 30 & 0 & 0 & 1 \\
\hline BAP entre 1 et 2 & 5 & 5 & 1 & 10 \\
\hline BAP entre 2 et 5 & 11 & 11 & 11 & 5 \\
\hline BAP entre 5 et 10 & 5 & 5 & 5 & 11 \\
\hline BAP entre 10 et 35 & 11 & 11 & 11 & 11 \\
\hline
\end{tabular}

Afin de prendre en considération la fidélité des méthodes analytiques et le domaine de concentration pour lequel la corrélation n'est pas très fiable, notre avis pour le contrôle industriel serait de tolérer une limite de $2 \mu \mathrm{g} / \mathrm{kg}$ pour le benzo[a]pyrène dans les huiles raffinées. Lorsque la teneur en BaP est inférieure à $2 \mu \mathrm{g} / \mathrm{kg}$, l'huile raffinée pourrait être jugée de qualité acceptable en considérant la limite de $5 \mu \mathrm{g} / \mathrm{kg}$ fixée par FEDIOL pour les hydrocarbures aromatiques polycycliques lourds. 


\section{Conclusion}

Les hydrocarbures aromatiques polycycliques sont des composés toxiques, notamment formés durant le séchage de matières premières telles que les pépins de raisin ou le coprah avant l'extraction de l'huile. Dans l'attente d'une réglementation européenne en préparation, le code des bonnes pratiques de FEDIOL devrait être suivi. II n'existe pas encore de méthode d'analyse normalisée pour l'analyse des HAP totaux. Un projet de commission concernant l'analyse de 12 HAP a été proposé par I'ITERG en 2001 à I'ISO/TC34/SC11 pour être évalué au niveau international. Cette méthode initialement développée pour les huiles de pépins de raisin et les huiles de coprah, peut être également utilisée pour d'autre huiles végétales telles que les huiles de colza, tournesol, soja et olive. Cependant, dans le cas des huiles de palme ou de grignons d'olive, les interférences liées à la complexité de la matrice ne permettent pas une analyse correcte par cette méthode. L'étude de l'effet du raffinage sur la teneur en HAP d'huiles brutes de pépins de raisin montre que les HAP lourds sont tous éliminés lors de l'étape de décoloration en présence de charbon actif alors que les HAP légers sont éliminés lors de la décoloration et de la désodorisation. L'analyse d'une quarantaine d'huiles raffinées commercialisées en France prouve qu'environ $20 \%$ d'entre elles ne respectent pas la limite fixée à $5 \mu \mathrm{g} / \mathrm{kg}$ par FEDIOL pour la teneur en HAP lourds. Basée sur plus de 60 résultats obtenus pour des huiles brutes ou raffinées, la corrélation entre la teneur en benzo[a]pyrène et les hydrocarbures aromatiques polycycliques lourds est bien meilleure que celle entre le BaP et les HAP totaux. L'analyse de la totalité des HAP étant une opération contraignante, la corrélation entre le benzo[a]pyrène et les hydrocarbures polycycliques lourds nous paraît suffisamment fiable pour que le contrôle des huiles en fin de raffinage soit réalisé en se basant sur l'analyse du BaP seul. Cependant, cette corrélation présentant une faiblesse dans le domaine de concentration de 1 à $2 \mu \mathrm{g} / \mathrm{kg}$ en benzo[a]pyrène, il serait plus prudent de considérer une limite maximale de $2 \mu \mathrm{g} / \mathrm{kg}$. Cette valeur pourrait être également conseillée lors du choix d'une limite au niveau européen, afin de tenir compte de la précision de la méthode d'analyse.

Ce travail a reçu le soutien de I'ACTIA (Association de Coordination Technique pour I'Industrie Agroalimentaire).

\section{RÉFÉRENCES}

1. US EPA. Review and evaluation of the evidence for cancer associated with air pollution, EPA-450/583-006R, U.S. Environmental Protection Agency, Arlington 1984.

2. DENNIS MJ, MASSEY RC, MCWEENY DJ, KNOWLES ME, WATSON D. Analysis of polycyclic aromatic hydrocarbons content in cereals, fats and other food products, Food Additives and Contaminants $1983 ; 8: 517-30$.

3. DE VOS RH, VAN DOKKUM W, SCHOUTEN A, DE JONG-BERKHOUT P. Polycyclic aromatic hydrocarbons in Dutch total diet samples (1984-1986), Food and Chemical Toxicology $1990 ; 28$ : 2638.

4. KAZEROUNI N, SINHA R, HSU CH, GREENBERG A, ROTHMAN N. Analysis of 200 food items for benzo[ $a]$ pyrene and estimation of its intake in an epidemiologic study, Food and Chemical Toxicology $2001 ; 39$ : 423-36. 
5. EUROPEAN COMMISSION, Opinion of the Scientific Committee on Food on the risks to human health of polycyclic aromatic hydrocarbons, Health and Consumer Protection Directorate General, 4 December 2002 (http://europa.eu.int/comm/food/fs/sc/scf/index_en.ht/m)

6. IARC. Polynuclear aromatic compounds. Part 1: Chemical environmental and experimental data. IARC Monographs on the evaluation of carcinogenic risks of chemicals to humans. Volume 32, International Agency for Research on Cancer, World Health Organization, Lyon 1983.

7. NISBET IC, LAGOY PK. Toxic equivalent factors for polycyclic aromatic hydrocarbons, Regulatory toxicology and pharmacology $1992 ; 16: 290-300$.

8. ISO 15302. Corps gras d'origines animale et végétale. Détermination de la teneur en benzo[a]pyrène. Méthode par chromatographie liquide haute performance en phase inverse 1998.

9. VIGNERON PY, ALLAERT JP, STOCLIN B. Determination of polycyclic aromatic hydrocarbons in vegetable oils: proposal for industrial quality control, Oléagineux Lipides Corps Gras (OCL) $2003 ; 10$ (2) : 155-8.

10. MORET S, CONTE LS. A rapid method for polycyclic aromatic hydrocarbons determination in vegetable oils, Journal of Separation Sciences $2002 ; 25: 96-100$.

11. MOREDA W, RODRIGUEZ-ACUNA R, PEREZ-CAMINO MC, CERT A. Determination of heavy polycyclic aromatic hydrocarbons in refined pomace olive oils, Instituto de la Grasa (CSIC), Document provided at 2002 Chemists Meeting of International Olive Oil Council (IOOC) 2002.

12. FIUME F, FERRIERI F, FROIO G, SPINELLI S, LATTARULO O, FANUZZI G. Determinazione di idrocarburi policiclici aromatici in oli alimentari, La Rivista Italiana delle Sostanze Grasse $2002 ; 79$ : 151-5.

13. VAN STIJN F, KERKHOFF MAT, VANDEGINSTE BGM. Determination of polycyclic aromatic hydrocarbons in edible oils and fats by on-line donor-acceptor complex chromatography and highperformance liquid chromatography with fluorescence detection, Journal of Chromatography $A$ $1996 ; 750: 263-73$.

14. RAOUX R, COUSTILLE JL, RAMBAUB S. Analyse des pesticides organochlorés, organophosphorés et des polychlorobiphényles dans les corps gras. Méthodes d'extraction et de purification multicartouches, Oléagineux Lipides Corps Gras (OCL) 197 ; 4 (1) : 71-80.

15. ISO/CD 15753. Animal and vegetable fats and oils. Determination of polycyclic aromatic hydrocarbons (Committee Draft) 2001.

16. HORWITZ W. Evaluation of Analytical Methods Used for Regulation of Foods and Drugs, Analytical Chemistry $1982 ; 54$ (1) : 67 A-76 A. $n$ 


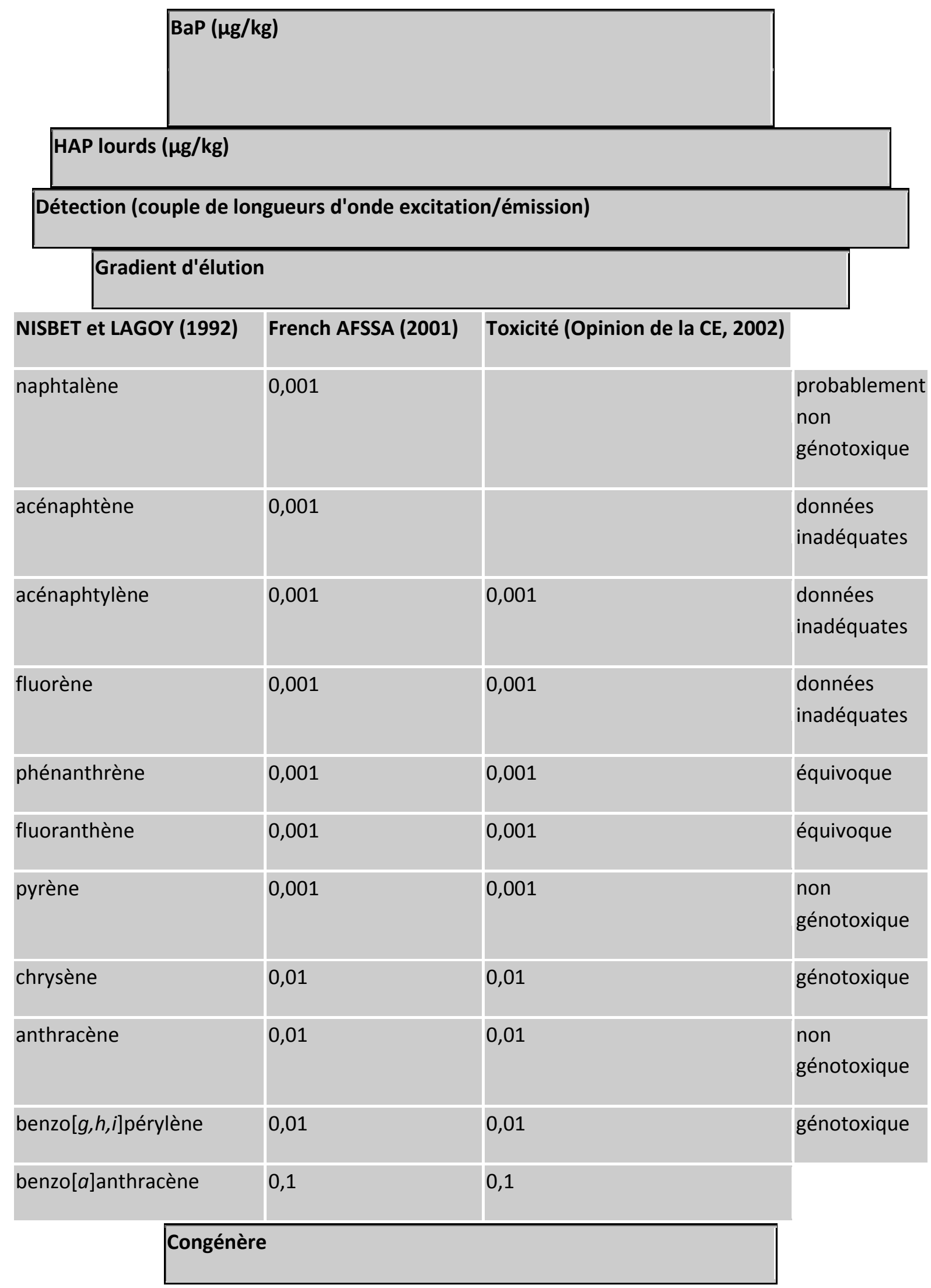




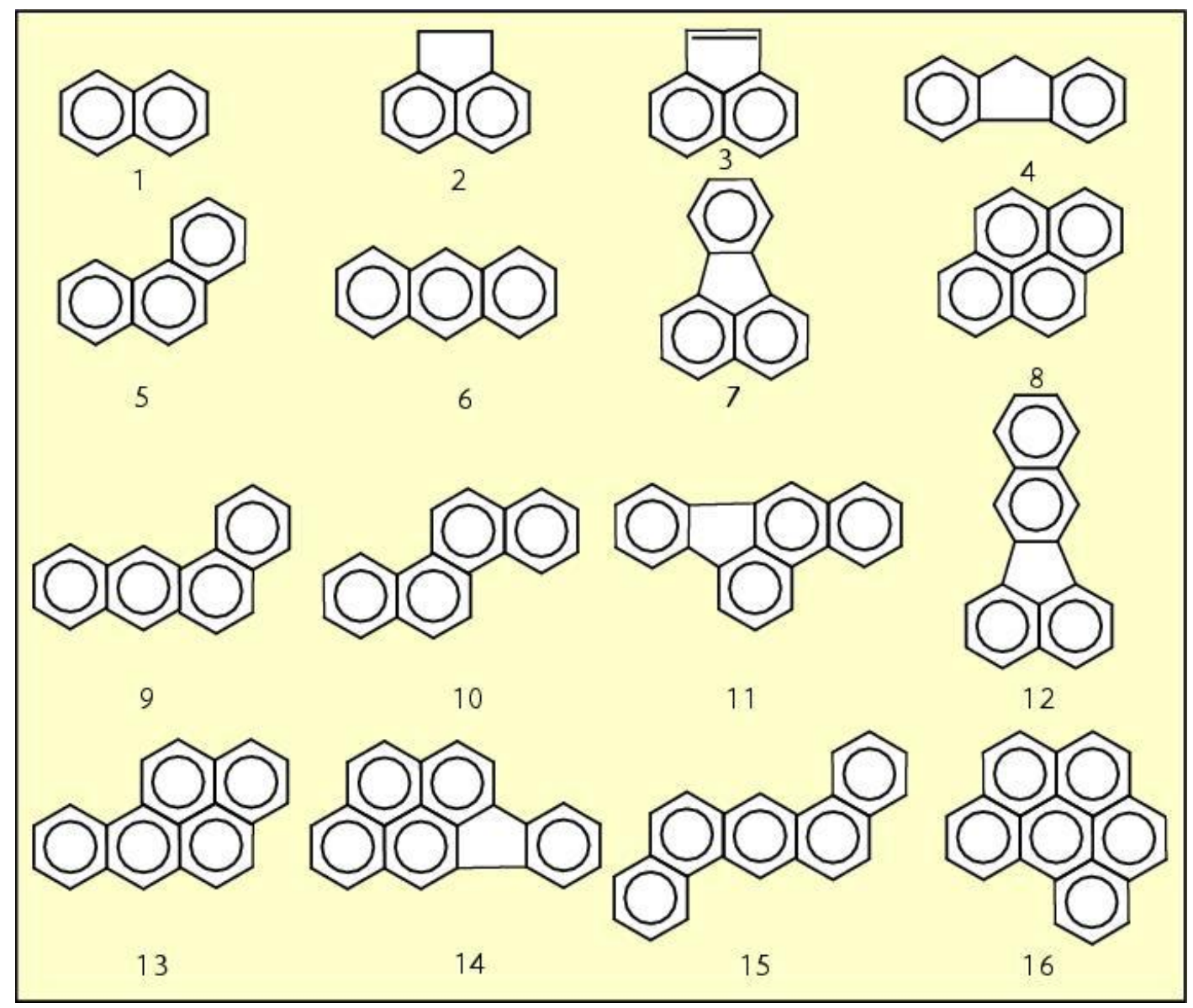

Tableau 1. Structure chimique des hydrocarbures polycycliques aromatiques.

(1) Naphtalène (NA), (2) Acénaphthène ( $A C)$, (3) Acénaphthylène ( $A C L)$, (4) Fluorène (FL), (5) Phénanthrène (PHE), (6) Anthracène (ATR), (7) Fluoranthène (FA), (8) Pyrène (PY), (9) Benzo[a]anthracène (BaA), (10) Chrysène (CHR), (11) Benzo[b]fluoranthène (BbFA), (12) Benzo[k]fluoranthène (BkFA), (13) Benzo[a]pyrène (BaP), (14) Indéno[1,2,3-cd]pyrène (IP), (15) Dibenzo[a,h]anthracène (DBahA), (16) Benzo[g,h,i]pérylène (BghiP). 


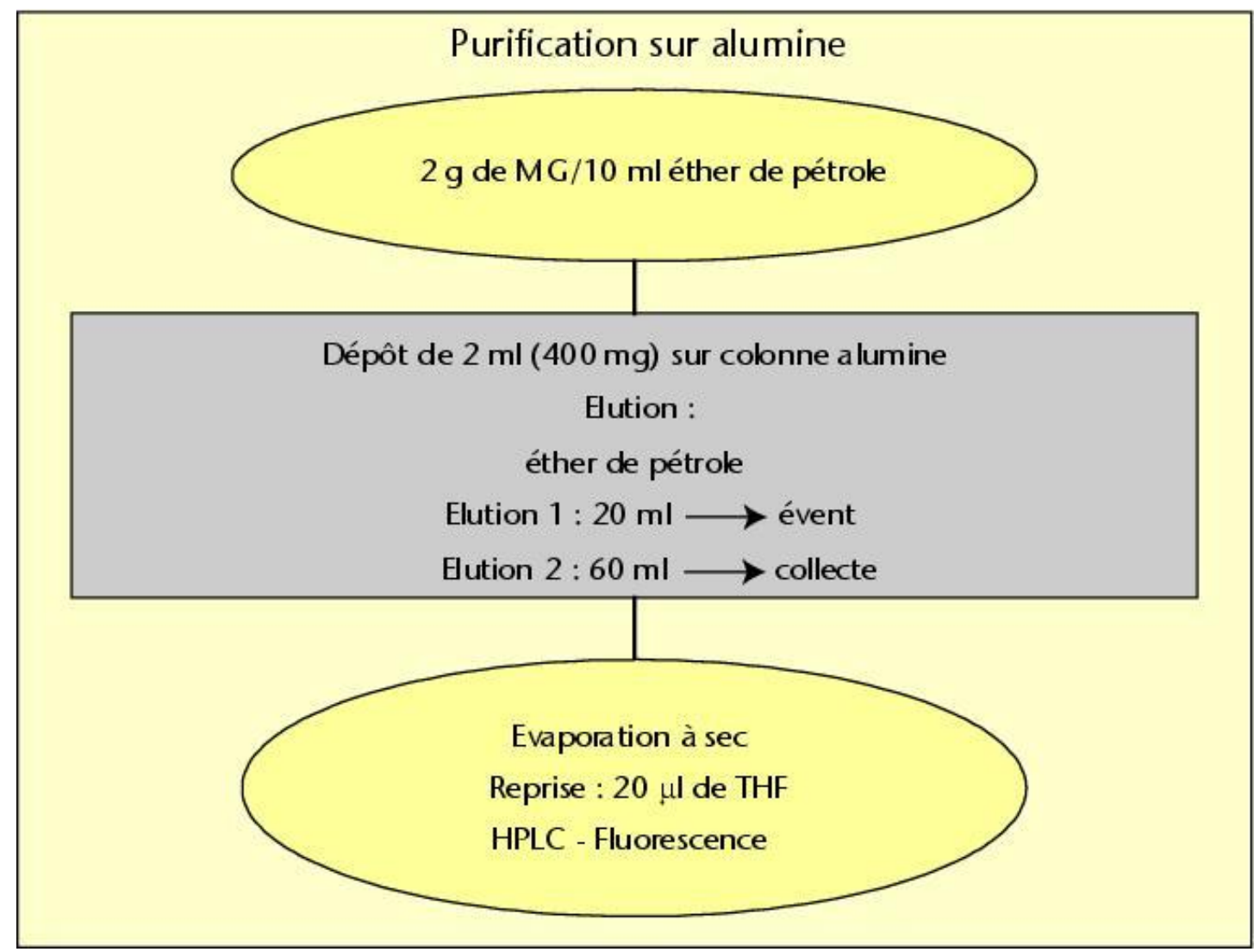

Figure 1. Protocole ISO 15302 pour le dosage du benzo[a]pyrène dans les huiles végétales.

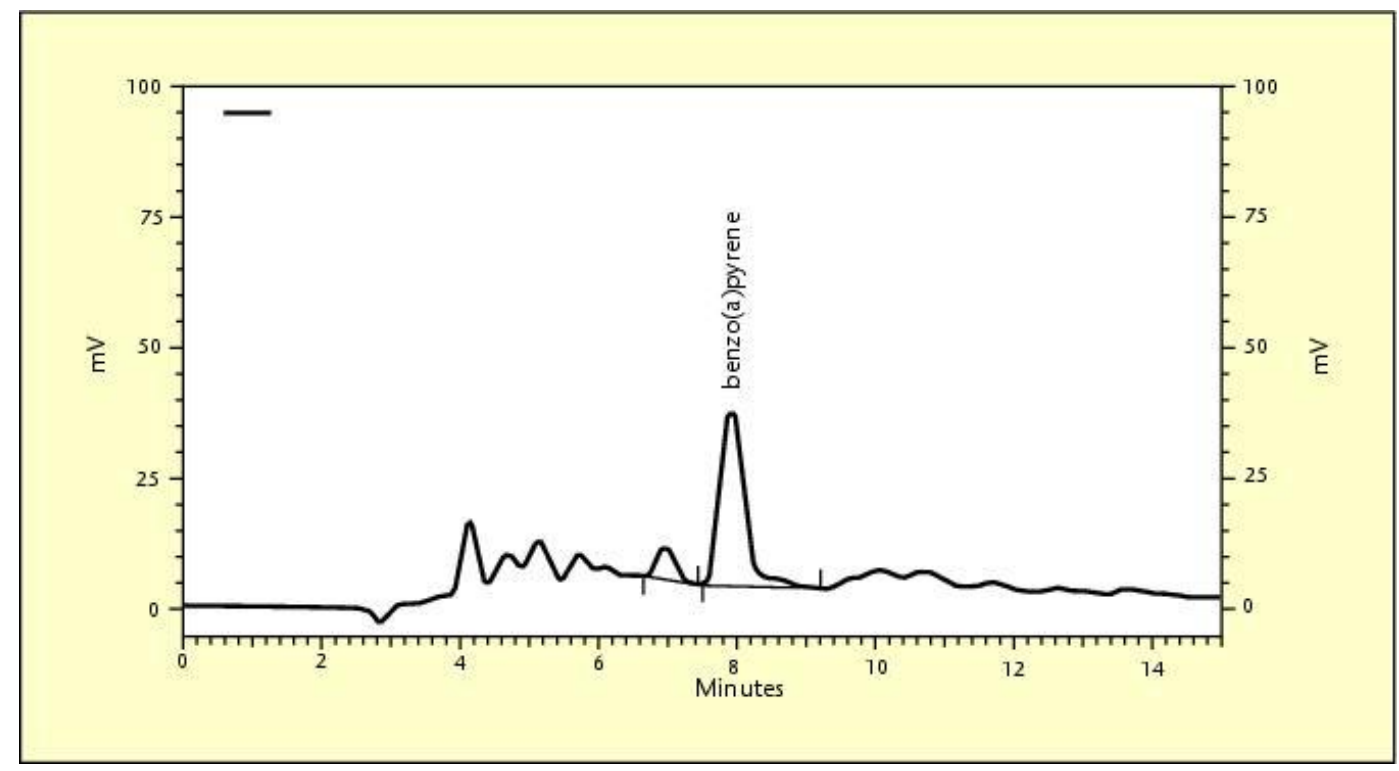

Figure 2. Chromatogramme de $7 \mu \mathrm{g} / \mathrm{kg}$ de benzo[a]pyrène dans une huile végétale raffinée selon la norme 15302. 


\section{Etape 1: Préconcentration des HAP dans la matière grasse}

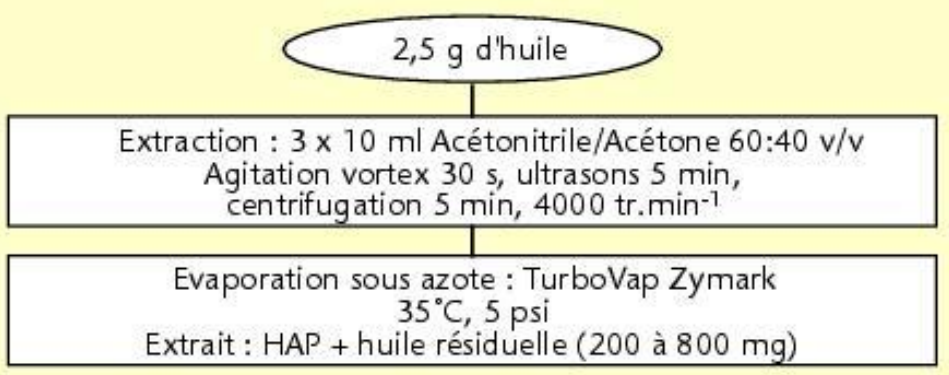

\section{Etape 2 : $\quad$ Extraction des HAP de I'huile résiduelle}

3 extractions successives :

$3 \times 2 \mathrm{ml}$ Acétonitrile/Acétone $60: 40 \mathrm{v} / \mathrm{v}$

Agitation vortex $10 \mathrm{~s}$, centrifugation 3 surnageants

\section{Etape 3 : Purification des HAP : deux extractions solide-liquide}

1 ère purification : cartouche $\mathrm{C18} / 2 \mathrm{~g}$

Conditionnement : $24 \mathrm{ml}$ Méthanol + $24 \mathrm{ml}$ Acétonitrile

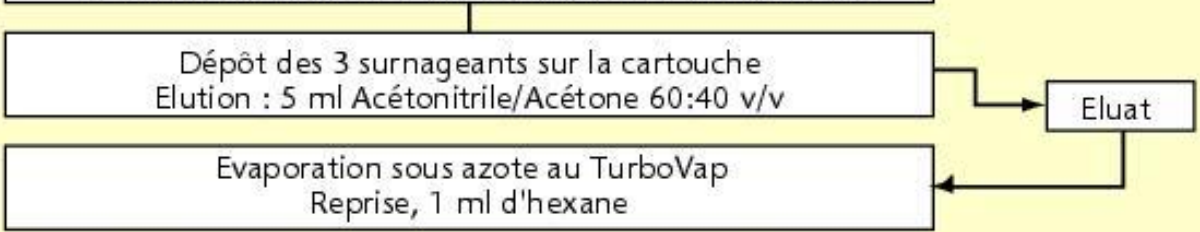

$2^{\text {ème }}$ purification : cartouche Florisil/500mg

Conditionnement : $15 \mathrm{ml}$ Dichlorométhane $+12 \mathrm{ml}$ Hexane

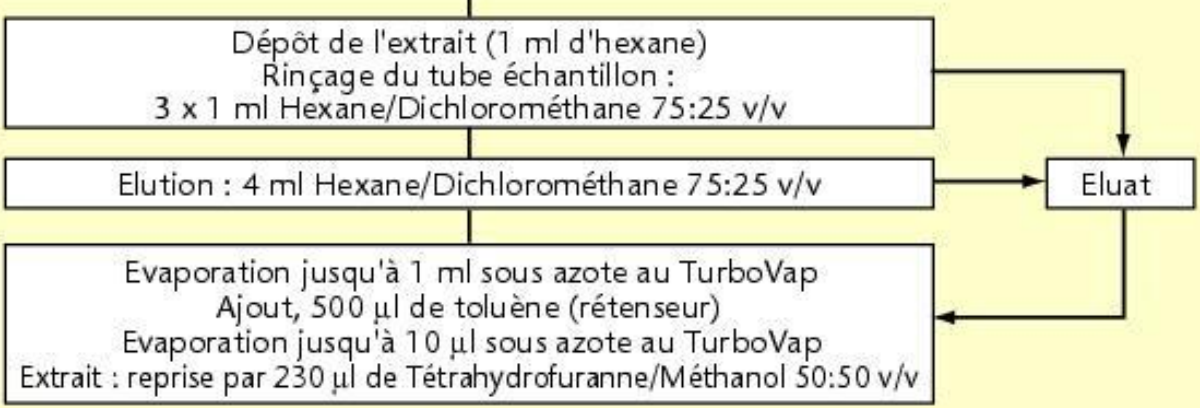

\section{Etape 4: Analyse des extraits}

HPLC - Fluorescence

Taux de récupération - Teneur en HAP des échantillons

Figure 3A. Protocole développé par l'Iterg pour la détermination des hydrocarbures polycycliques aromatiques dans les huiles végétales (ISO/CD 15753 : 2001). Méthode générale. 


\section{Etape 1: Préconcentration des HAP dans la matière grasse}

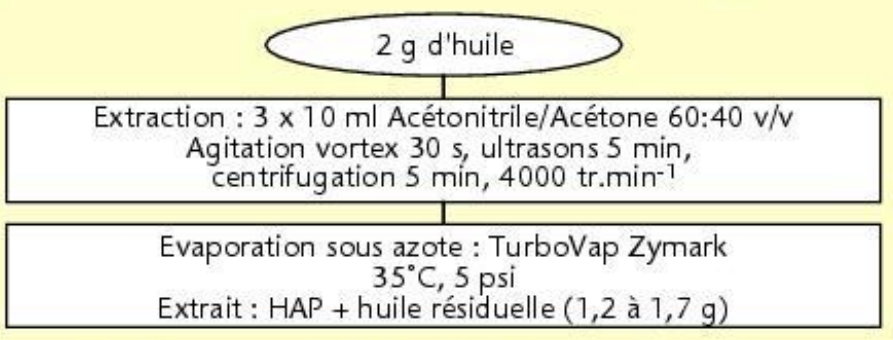

Etape 2 : 2ème préoncentration des HAP dans la matière grasse

3 extractions successives:
$3 \times 2 \mathrm{ml}$ Acétonitrile/Acétone $60: 40 \mathrm{v} / \mathrm{v}$
Agitation vortex 10 s, centrifugation
3 surnageants : évaporation sous azote au TurboVap
Extrait: HAP + matière grasse résiduelle
Division de l'extrait en deux

Etape 3 : Purification des HAP : deux extractions solide-liquide $1^{\text {ère }}$ purification : 2 cartouches $\mathrm{C18} / 2 \mathrm{~g}$

Conditionnement : $24 \mathrm{ml}$ Méthanol $+24 \mathrm{ml}$ Acétonitrile

Dépôt de l'extrait sur 2 cartouches et rinçage des tubes :

$3 \times 2 \mathrm{ml}$ Acétonitrile/Acétone $80: 20 \mathrm{v} / \mathrm{v}$

Elution : $5 \mathrm{ml}$ Acétonitrile/Acétone $80: 20 \mathrm{v} / \mathrm{v}$

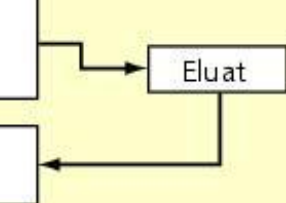

Evaporation sous azote au TurboVap Reprise, $1 \mathrm{ml} \mathrm{d}$ 'hexane

$2^{\text {ème }}$ purification : 2 cartouche Florisil/500 mg

Conditionnement : $15 \mathrm{ml}$ Dichlorométhane $+12 \mathrm{ml}$ Hexane

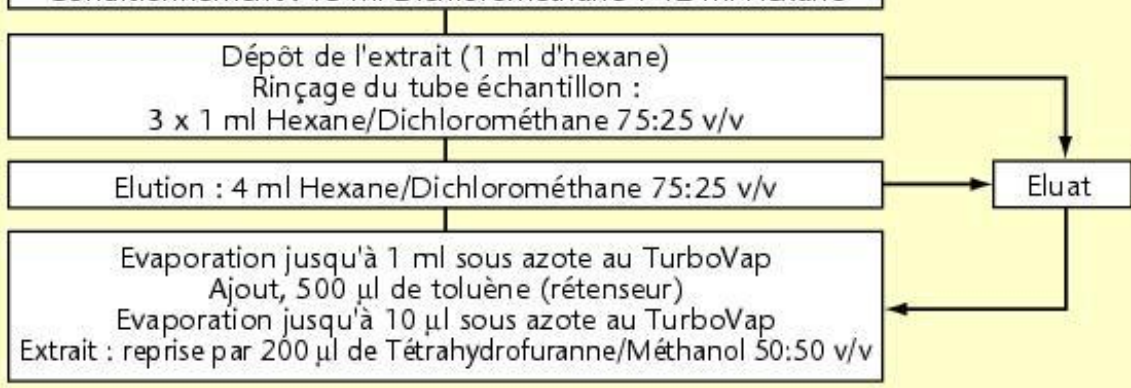

Etape 4 :

\section{Analyse des extraits}

HPLC - Fluorescence
Taux de récupération - Teneur en HAP des échantillons

Figure 3B. Procédure développée par I"Iterg pour la détermination des hydrocarbures polycycliques aromatiques dans les huiles végétales (ISO/CD 15753 : 2001). Méthode spécifique pour I'huile de coprah. 


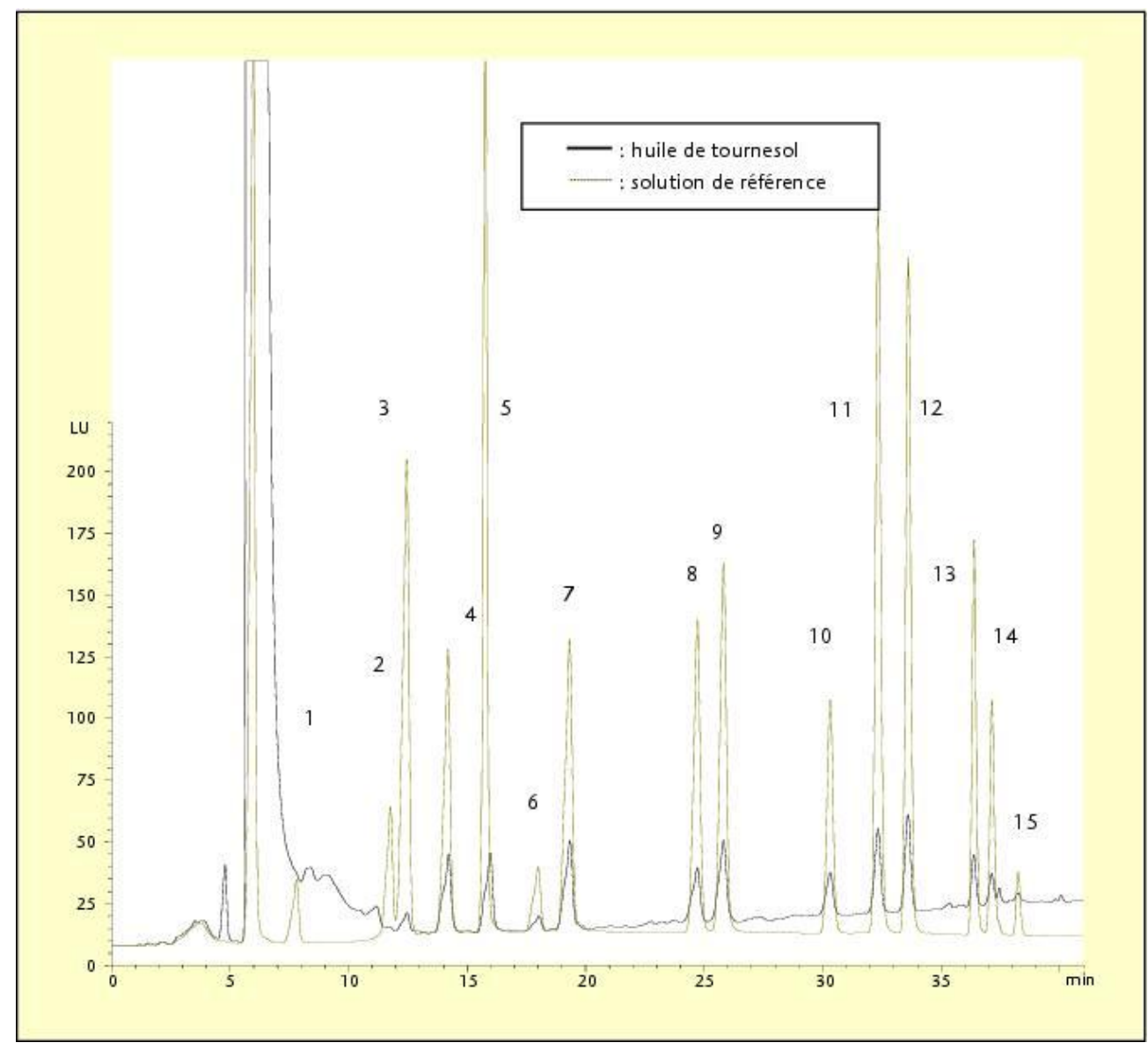

Figure 4. Chromatogramme des hydrocarbures polycycliques aromatiques selon la méthode de Iterg: (A) huile de tournesol raffinée complémentée avec $1 \mu \mathrm{g} / \mathrm{kg}$ de HAP (B) solution de référence $(5 \mu \mathrm{g} / \mathrm{kg})$. (1) Naphtalène (NA), (2) Acénaphtène (AC), (3) Fluorène (FL), (4) Phénanthrène (PHE), (5) Anthracène (ATR), (6) Fluoranthène (FA), (7) Pyrène (PY), (8) Benzo[a]anthracène (BaA), (9) Chrysène (CHR), (10) Benzo[b]fluoranthène (BbFA), (11) Benzo[k]fluoranthène (BkFA), (12) Benzo[a]pyrène (BaP), (13) Dibenzo[a,h]anthracène (DBahA), (14) Benzo[g,h,i]pérylène (BghiP), (15) Indéno[1,2,3-cd]pyrène (IP). 


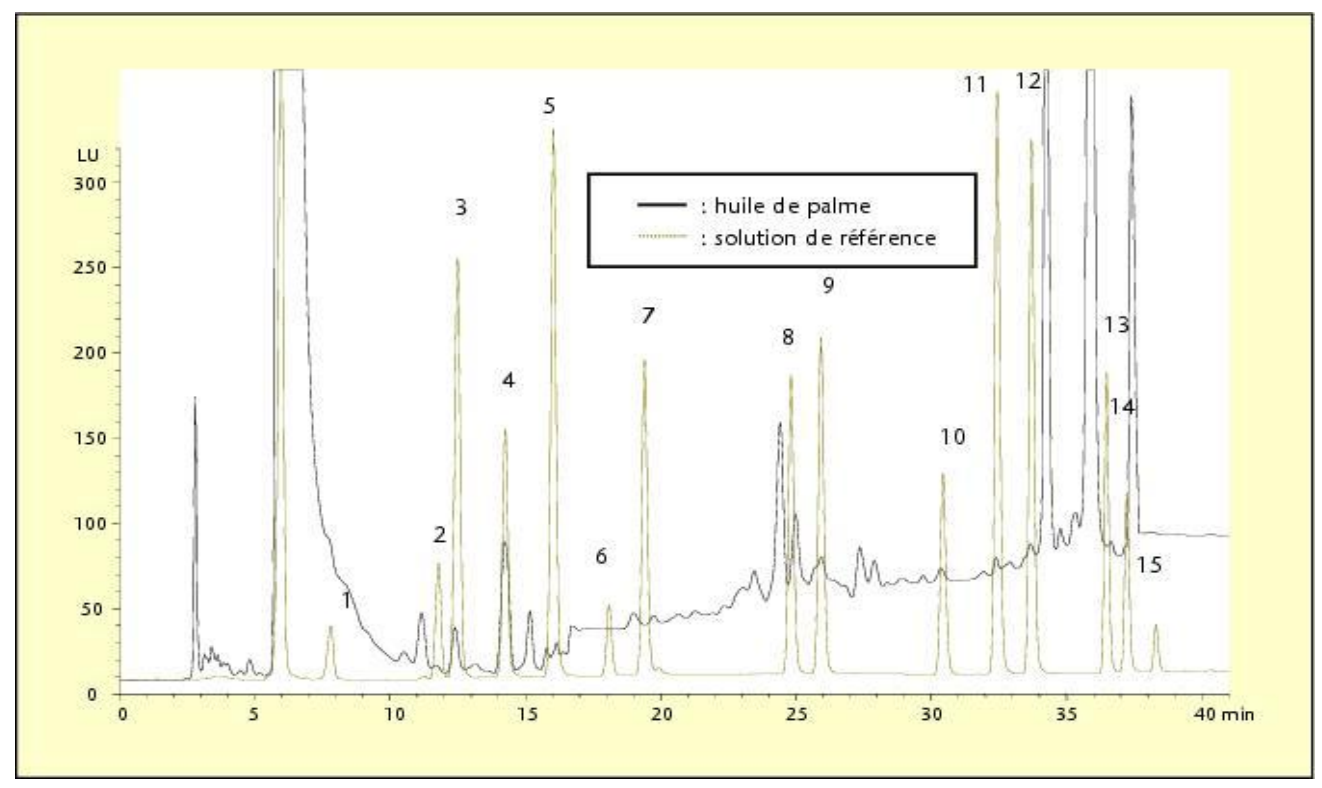

Figure 5. Chromatogramme des hydrocarbures polycycliques aromatiques selon la méthode Iterg : (A) huile de palme raffinée (B) solution de référence $(5 \mu \mathrm{g} / \mathrm{kg})$

(1) Naphtalène (NA), (2) Acénaphtène (AC), (3) Fluorène (FL), (4) Phénanthrène (PHE), (5) Anthracène (ATR), (6) Fluoranthène (FA), (7) Pyrène (PY), (8) Benzo[a]anthracène (BaA), (9) Chrysène (CHR), (10) Benzo[b]fluoranthène (BbFA), (11) Benzo[k]fluoranthène (BkFA), (12) Benzo[a]pyrène (BaP), (13) Dibenzo[a,h]anthracène (DBahA), (14) Benzo[g,h,i]pérylène (BghiP), (15) Indéno[1,2,3-cd]pyrène (IP).

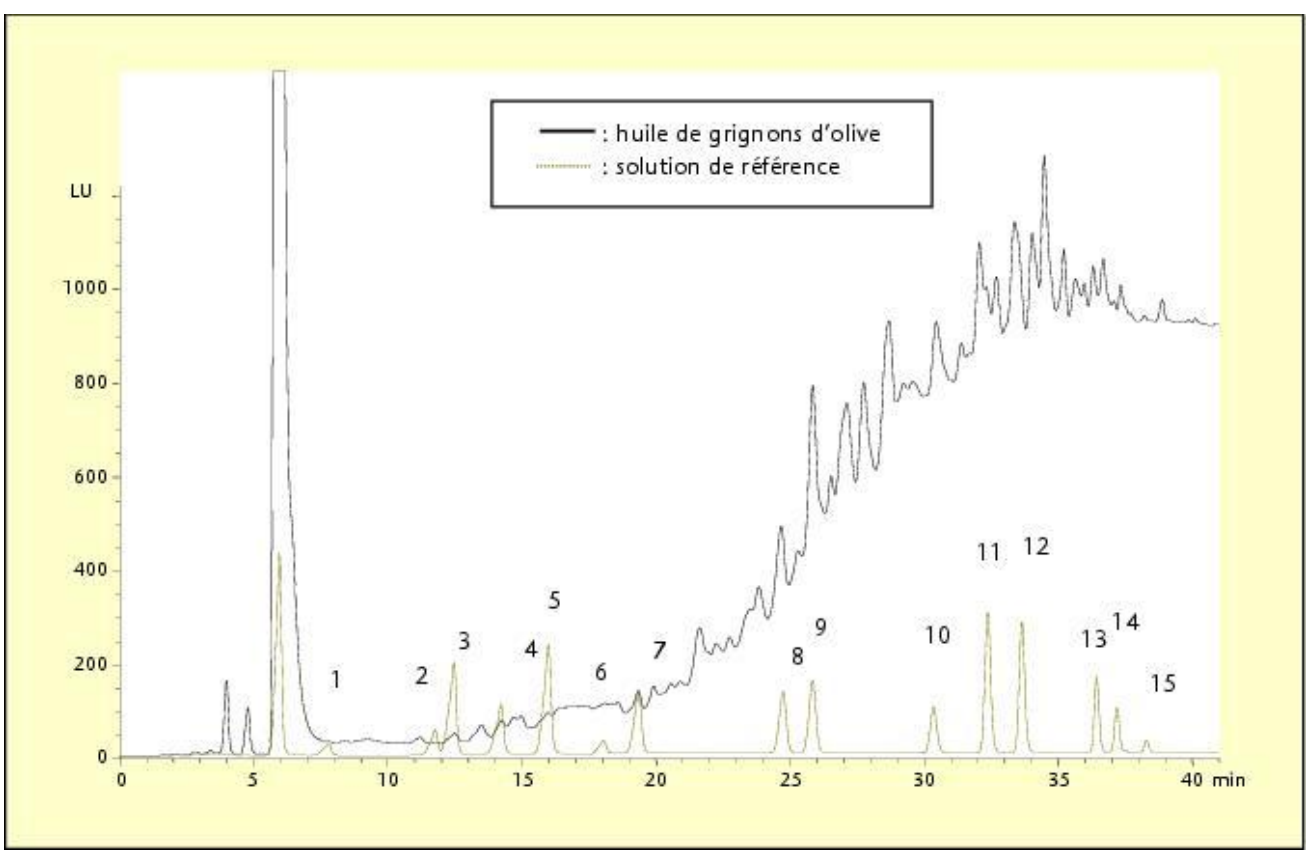

Figure 6. Chromatogramme des hydrocarbures polycycliques aromatiques selon la procédure Iterg : (A) huile de grignons d'olive raffinée (B) solution de référence $(5 \mu \mathrm{g} / \mathrm{kg})$ (1) Naphtalène (NA), (2) Acénaphtène (AC), (3) Fluorène (FL), (4) Phénanthrène (PHE), (5) Anthracène (ATR), (6) Fluoranthène (FA), (7) Pyrène (PY), (8) Benzo[a]anthracène (BaA), (9) Chrysène (CHR), (10) Benzo[b]fluoranthène (BbFA), (11) Benzo[k]fluoranthène (BkFA), (12) Benzo[a]pyrène (BaP), (13) Dibenzo[a,h]anthracène (DBahA), (14) Benzo[g,h,i]pérylène (BghiP), (15) Indéno[1,2,3-cd]pyrène (IP). 


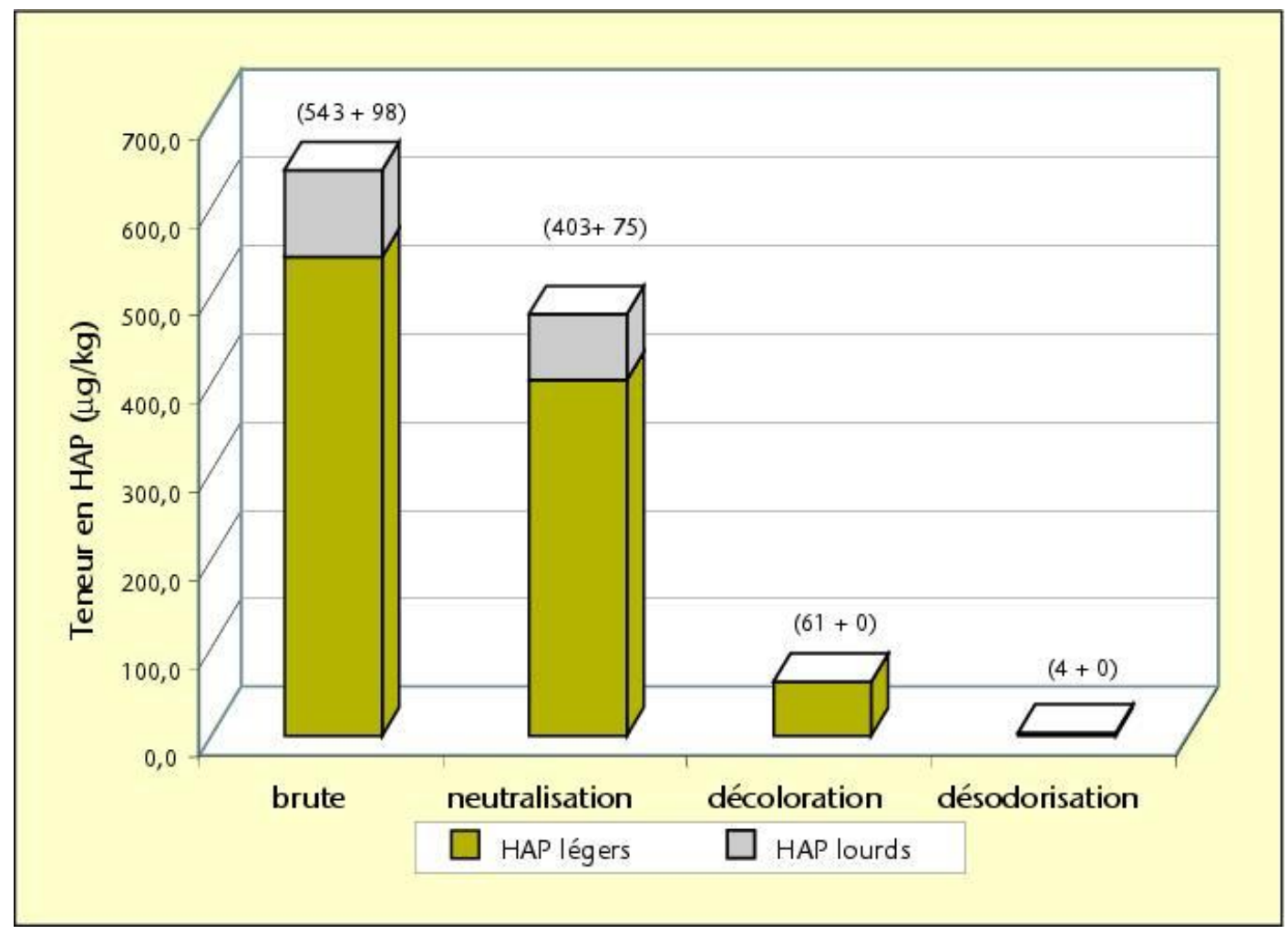

Figure 7. Elimination des hydrocarbures aromatiques polycycliques des huiles de pépins de raisin durant le raffinage (moyenne de trois échantillons).

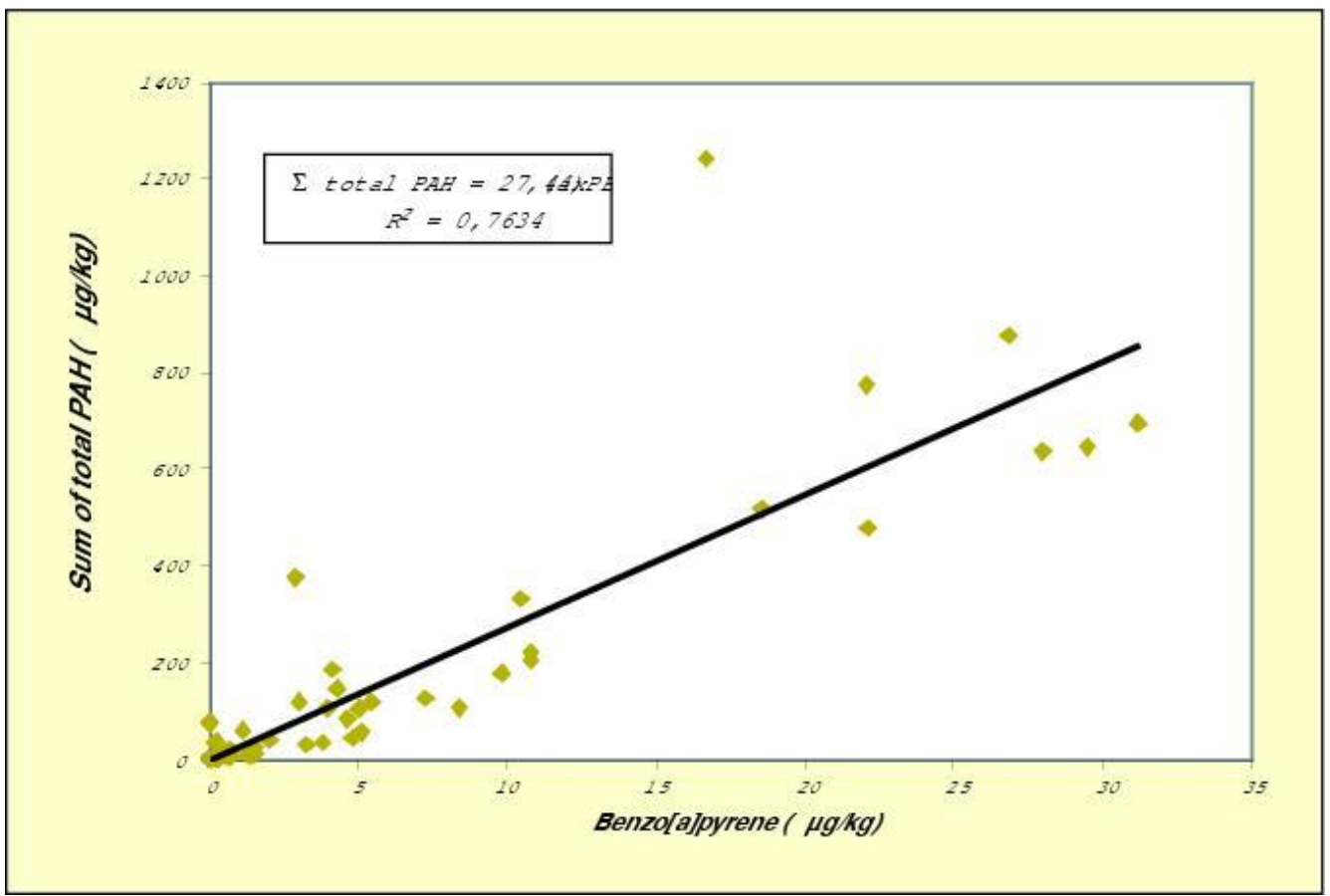

Figure 8. Corrélation entre la teneur en benzo[a]pyrène et la teneur en hydrocarbures aromatiques polycycliques totaux mesurées par la méthode de l'Iterg (résultats de 62 huiles végétales brutes et raffinées). 


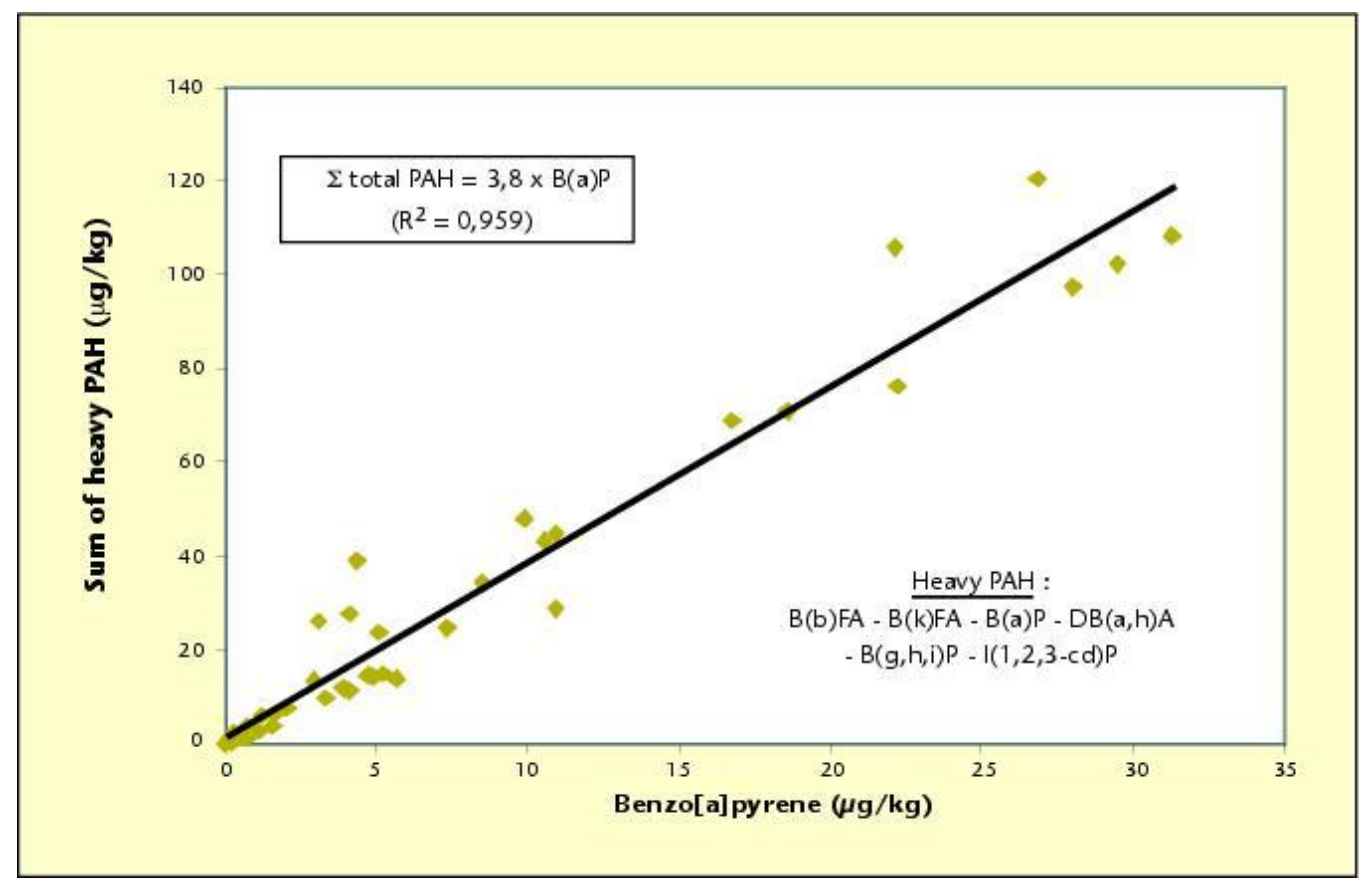

Figure 9. Corrélation entre la teneur en benzo[a]pyrène et la teneur en hydrocarbures aromatiques polycycliques lourds mesurées par la méthode de l'Iterg (résultats de 62 huiles végétales brutes et raffinées). 